\title{
Hacia una didáctica del sentido. Didácticas emergentes de las prácticas pedagógicas de filosofía en tres escuelas católicas'
}

\author{
Johan Andrés Nieto Bravo ${ }^{2}$
}

Recibido: 02-02-2017

Aceptado: 14-04-2017

\section{RESUMEN}

Ante la poca claridad del papel que ejerce la filosofía como aprendizaje escolar, la presente investigación buscó identificar las didácticas que subyacen de las prácticas pedagógicas en filosofía, a fin de analizar la problemática didáctica del enseñar filosofía o enseñar a filosofar. El enfoque cualitativo de ejercicio investigativo, ha propiciado una aproximación al contexto de tres instituciones educativas católicas y a las comprensiones de estudiantes y docentes que comparten el Espacio Académico de Filosofía. La perspectiva epistemológica hermenéutica, concilia las relaciones de distancia entre los protagonistas que se encuentran en el aula filosófica, a fin de proponer una didáctica del sentido, en donde los significados de la filosofía, se encuentre con los significantes, es decir con los filósofos estudiantes, a fin de construir un conocimiento con sentido. El presente texto presenta el desarrollo de la primera categoría de la investigación, a la cual se le denomina didáctica de la filosofía.

Palabras clave: escuela católica, didáctica, filosofía, práctica pedagógica, sentido.

1. La presente investigación hace parte de la Línea Medular Enrique Lacordaire, sobre educación y libertades, a la cual tributa la Vicerrectoría de Universidad Abierta y a Distancia en su campo interdisciplinar de Pedagogía, currículo, didáctica y evaluación, desde el cual se fundamenta la línea activa de la Facultad de Educación sobre Pedagogía, Currículo y evaluación, en la que se fundamenta la línea de programa Pedagogía de la Filosofía, desde la cual se desarrolló este ejercicio investigativo.

2. Licenciado en Filosofía y Educación Religiosa de la Universidad Santo Tomás, docente investigador de la Facultad de Educación de la Vicerrectoría de Universidad Abierta y a Distancia de la Universidad Santo Tomás. Perteneciente como docente de tiempo completo de la Licenciatura en Filosofía, Pensamiento Político y Económico. Maestrante en Educación.

Correo electrónico: johannieto@ustadistancia.edu.co

ORCID: 0000-0002-8608-8511 


\title{
Towards a didactics of meaning. Emerging didactics of pedagogical practices of philosophy in three Catholic schools
}

\begin{abstract}
Given the lack of clarity exerted by philosophy as scholar learning, this investigation look for identify the underlying pedagogical practices in philosophy, in order to analize the didactic problems of teaching philosophy or teaching philosophizing. The qualitative aproach of the investigative exercise, has made possible an approach to three catholic schools contexts, and to the comprehension of students and teachers that share a philosophical academical space. The epistemological and hermeneutical perspective, conciliates the distant relationships among protagonists that are at the philosophical classroom, in order to propose a didactic of sense, where the significance of philosophy, meet whith the signifiers, in other words with the philosophers students, to built a full of sense knowledge. This text shows the first category of investigations developement, which names philosophy's didact.
\end{abstract}

Keywords: Catholic School, Didactic, Philoslophy, Pedagogical practice, Sense.

\section{Introducción}

La enseñanza de la filosofía en la escuela secundaria colombiana, ha tenido una serie de transformaciones desde la promulgación de la Ley General de Educación 115 de 1994, la cual dejó sin un sitio preponderante dentro del currículo a este Espacio Académico que era considerado por la anterior legislación como un aprendizaje de capital importancia. La no existencia de unos lineamientos curriculares oficiales ${ }^{3}$, dificulta la calidad y la supervisión de un ideal filosófico en cuanto enseñanza en el ámbito 
escolar, lo cual ha producido como resultado la instrumentalización de esta materia y la multiplicidad de posibilidades de orden pragmático que se le da a este espacio.

Ante esta realidad que atañe al aprendizaje de filosofía en la escuela secundaria, se problematizó el sentido de la enseñanza de la filosofía en el bachillerato, su "¿para qué?". Esta problematización dio por resultado un horizonte de finalidad, desde el cual se reflexiona alrededor de la didáctica filosófica como una ciencia analógica que orienta las prácticas pedagógicas de los docentes de filosofía en la escuela, unificando un criterio de importancia curricular en la formación filosófica de los estudiantes de bachillerato, como una producción aprendida, pero también como una producción construida por el mismo estudiante.

Los estudios académicos e investigativos desarrollados alrededor de la relación entre didáctica y filosofía, han coincidido en la necesidad de diferenciar los objetos de estudio de ambas ciencias, sin querer paragonar o subordinar la didáctica a la filosofía misma. Alrededor de esta problemática se han desarrollado diferentes investigaciones. Por su parte Alejandro Cerletti ha desarrollado una problematización en torno a las mediaciones didácticas que posibilitan la relación entre el docente y el estudiante de filosofía; de igual forma Alfonso Ciprian reflexiona sobre los impedimentos que dificultan el aprendizaje significativo de la filosofía escolar, en torno a los actores del proceso de enseñanza aprendizaje. Desde una mirada a la educación Latinoamericana Ricardo Navia expone sistemáticamente las condiciones sociales que rodean el entorno del aprendizaje como un condicional significativo para el desarrollo del mismo, concluyendo que la enseñanza de la filosofía es sensible a la condición del pueblo latinoamericano,

Preguntarse por las didácticas filosóficas a partir de las prácticas pedagógicas desarrolladas en la escuela católica, permite a esta investigación delimitar el tema y por ende orientar la reflexión hacia un horizonte de transformación de las prácticas pedagógicas mismas. A partir de este horizonte, la investigación es desarrollada en el contexto escolar de tres instituciones pertenecientes al Sistema Educativo Arquidiocesano y de los conceptos teóricos desarrollados entorno a la problemática, a fin de estudiar las didácticas que subyacen de las prácticas pedagógicas en filosofía dentro del contexto de la escuela católica. Han sido tres las categorías consolidadas: Didáctica de la Filosofía; Práctica pedagógica y Escuela Católica, las cuales fueron abordadas desde 
diferentes autores, que entran en diálogo con el proceso reflexivo del autor a fin de comprender el fenómeno estudiado.

Este documento expone en un primer momento el sistema metodológico adoptado por la investigación, el cual asumió un enfoque cualitativo, que analiza los datos desde una perspectiva epistemológica hermenéutica, optando por una etnografía educativa, cuyos instrumentos de recolección de datos apropian la perspectiva del docente y de los estudiantes, de cara a las didácticas filosóficas que se generan en las prácticas pedagógicas en filosofía; en segundo lugar se presenta la interpretación y discusión de los datos recolectados en las entrevistas a los docentes y el grupo focal de cada institución, los cuales han permitido hacer un análisis a las didácticas filosóficas empleadas por los docentes en sus prácticas pedagógicas y el impacto generado en los estudiantes. Finalmente se concluye recogiendo los objetivos trazados por la investigación.

\section{Sistema metodológico}

En primer lugar, el enfoque investigativo en el cual se enmarca esta investigación, es el cualitativo, caracterizado por una metodología interpretativa, que parte de la inducción, es decir del encuentro con la realidad, la flexibilidad y sensibilidad que el investigador asume frente al contexto y a los sujetos conocidos. Este enfoque optimiza los procesos de diálogo e interacción con las prácticas pedagógicas docentes en filosofía, las cuales están compuestas por sujetos activos y cognoscentes, a partir de los cuales se puede fundamentar una teoría basada en el encuentro y la experiencia empírica.

La investigación cualitativa se interesa, en especial, por la forma en la que el mundo es comprendido, experimentado y producido; por el contexto y por los procesos; por la perspectiva de los participantes, por sus sentidos, pos sus significados, por su experiencia, por sus conocimientos, por sus relatos. (Vasilachis, 2006, p. 29)

En tal sentido el enfoque cualitativo adoptado por esta investigación, se centra en la comprensión de la problemática didáctica de la enseñanza de la filosofía en la escuela, partiendo de una realidad específica que es la 
práctica docente, la cual se encuentra sujeta a un proceso de comprensión, en el que se deben estudiar las líneas teóricas y prácticas, su concordancia y congruencia, a fin de dar respuesta a la pregunta problema aquí planteada. Durante el desarrollo de la investigación se estudia la forma en la que el docente de filosofía comprende, experimenta y produce el aprendizaje dentro del aula escolar, a partir de un acercamiento al contexto real de la práctica pedagógica en filosofía, a sus experiencias, significados, conocimientos y comprensiones.

En segundo lugar se encuentra la perspectiva epistemológica hermenéutica de esta investigación, la cual reconoce la presencia de dos sujetos en el proceso de comprensión epistemológica de la investigación cualitativa, por un lado se encuentra el "Sujeto Cognoscente" 4 y por otro lado el "Sujeto Conocido", entre ambos protagonistas de la investigación existe una distancia, la cual impide el mutuo conocimiento. La herramienta para conciliar dicha distancia es la interpretación como fundamento cualitativo de la investigación, la cual producirá una "metaepistemología, que supone la copresencia de dicha epistemología con la del sujeto cognoscente y la mutua complementación de ambas; el paradigma interpretativo sería entonces presupuesto de la investigación cualitativa" (Vasilachis, 2006, pp. 59-60).

Esta comprensión de la distancia existente entre los dos sujetos de conocimiento en la investigación, posibilita la implementación de una epistemología hermenéutica, la cual ponga en diálogo interpretativo la práctica pedagógica que se suscita dentro de las aulas de filosofía, y los presupuestos teóricos acuñados en esta investigación, los cuales problematizan dicha práctica y buscan teorías fundamentadas desde la experiencia empírica, que den respuesta a un proyecto de didáctica filosófica escolar.

La acción humana es como un texto, tiene en sí mismo una existencia propia, y está sujeto a múltiples interpretaciones según la distancia y la relación que ejerza con el intérprete, así entonces el actuar humano está abierto a las diversas interpretaciones que se generan gracias a la comprensión que se realice de éste. Del texto a la acción, de la acción al

4. El Sujeto Cognoscente, es una categoría empleada por Irene Vasilachis, para situar el dominio de una epistemología propia del investigador, "(...) a partir de ese sujeto se construyen, desarrollan, aplican, legitiman y cuestionan las distintas formas de conocimiento" (Vasilachis, 2006, p. 57).

5. El Sujeto Conocido, es una categoría empleada por Irene Vasilachis, para dar una identidad y existencia a los sujetos de la investigación, que posibilita la comprensión de los mismos, desde un criterio de igualdad. 
texto, y en el entramado de este diálogo, se encuentra la comprensión; sin embargo, el fenómeno del actuar humano, como también el del texto, poseen la dificultad del pluralismo-interpretativo, ante éste se conjetura y se valida, para encontrar la aproximación más apta. De allí que el acto hermenéutico supone un diálogo entre comprender y explicar:

La comprensión está totalmente mediatizada por el conjunto de los procedimientos explicativos que la preceden y la acompañan. La contrapartida de esta apropiación personal no es algo que se pueda sentir: es el significado dinámico que la explicación pone de manifiesto y que hemos identificado antes con la referencia del texto, es decir, con su capacidad de revelar el mundo. (Ricoeur, 2000, p. 194)

Al optar por esta perspectiva epistemológica, esta investigación se aproxima al campo de trabajo con la conciencia que los sujetos conocidos distan del investigador cognoscente, sin embargo, no son realidades estáticas, sino seres que al poseer una existencia propia, se encuentran en devenir, en transformación permanente y supeditados a múltiples formas de ser interpretados. Ante esta particularidad se asume la perspectiva de Ricoeur en la cual comprensión y explicación van de la mano, a fin de generar una interpretación más cercana a la realidad conocida, a su contexto y a la red simbólica de significaciones que la constituye.

En tercer lugar, esta ruta metodológica asume a la etnografía como el tipo de investigación, ya que esta comprende el trabajo del investigador como un describir el contexto, comprender los significados e interactuar con la cultura, haciendo parte de un proyecto insustituiblemente humano. Al ser un dinamismo humano supone dos dimensiones, por un lado, el encuentro con la realidad desde el trabajo de inmersión en el campo; por otro lado la reflexividad teórica que asume frente a las experiencias suscitadas por la práctica, así entonces la etnografía es un tipo de investigación que hace reflexiva la práctica:

Es una reflexión que conduce a una revisión acerca del modo y la forma en que los sujetos producen el conocimiento social imprescindible para la coexistencia en sociedad. El punto de partida de la reflexividad implica considerar así al hombre como parte del mundo social, interactuado, observando y participando con otros hombres en un contexto y en una situación espacio-temporal determinada y, desde allí, 
considerar al propio investigador como parte del mundo que estudia. (Ameigeiras, 2006, p. 115)

Esta investigación toma distancia de las visiones positivistas o las materialistas, las cuales generan una división entre teoría y práctica al interior del quehacer etnográfico, no vasta entonces con describir, es necesario teorizar, ya que la descripción de una realidad, supone su conceptualización. La línea directriz de esta investigación se encuentra en la analogía y el diálogo entre los presupuestos teóricos dados por la razón, y la producción práctica generada por la experiencia empírica. De allí que el investigador interactúa y se inserta en el campo de trabajo, pero no pasa por obvio los contenidos teóricos estudiados, sino que los confronta con la experiencia, a fin de suscitar teorías fundamentadas en una práctica reflexiva.

El diálogo entre la teoría y la práctica en el ejercicio etnográfico, le permite direccionarse hacia un tipo de etnografía específico, que es el de la etnografía educativa, el cual responde a los procesos de investigación que se suscitan dentro de las aulas de clase, como un espacio específico de formación humana, en donde interactúan los conocimientos producidos por la humanidad a lo largo de la historia, y los conocimientos producidos por los estudiantes en el aquí y el ahora, por ello "al adoptar la etnografía en el campo de investigación educativa, es importante no acceder a ella como una simple técnica, sino tratarla como una opción metodológica, en el entendido de que todo método implica teoría" (Rockwell, 1999, p. 25).

Esta investigación aplica la etnografía como una opción metodológica que se problematiza sobre el fenómeno social educativo que gira en torno al aprendizaje de la filosofía en la escuela, pero al mismo tiempo puntualiza dicho fenómeno en la especificidad de los contextos en los cuales se produce cada una de las prácticas docentes. La escuela es entonces vista desde sí misma en el micro-contexto del aula filosófica, pero al mismo tiempo confrontada con la teoría social que se aplica a esta disciplina pedagógica, desde los presupuestos del MEN. La etnografía educativa posibilita dicha confrontación y diálogo, entre el micro y el macro entorno social del aprendizaje filosófico en la escuela.

En cuarto lugar, se presentan las técnicas de recolección de datos, las cuales responden al tipo de investigación etnográfica y al enfoque cualitativo, dichas técnicas son la entrevista a docentes y el desarrollo 
de grupos focales con estudiantes. Con relación a la entrevista, ésta se constituye como una herramienta que supone la relación del sujeto cognoscente con el sujeto conocido, junto al contexto en el que se suscita la investigación. Esta herramienta supone la integración de los sujetos, sobre la cual se generarán diálogos con preguntas y respuestas, que producirán conocimiento, conclusiones y datos. Así entonces la finalidad de la entrevista es conocer la óptica desde la cual los maestros ven y asumen la práctica pedagógica docente en filosofía:

\section{Se trata de un tipo de entrevista que requiere de manera imprescindible de un ejercicio del diálogo sustentado a en una capacidad de escucha, que permite estar más atento a lo que el otro dice, expresa, sugiera, que a lo que al investigador le preocupa. (Ameigeiras, 2006, p. 129)}

El contacto con los diferentes profesores, produce el conocimiento de diferentes didácticas filosóficas, las cuales permitirán comprender el estado actual del aprendizaje en las instituciones escolares. Prima entonces en la entrevista el poder encontrar las perspectivas didácticas desde las cuales se direcciona la clase de filosofía, sobre la perspectiva didáctica del investigador, lo que no supone, que en el diálogo no se puedan confrontar ambas perspectivas.

En cuanto a la entrevista, se realizará una entrevista semi-estructurada, basada en un diálogo con cada docente orientador del aprendizaje de filosofía de los tres colegios. La entrevista tendrá tres momentos de diálogo, cada momento responde a una de las categorías componentes de esta investigación a saber: didáctica de la filosofía, práctica pedagógica y escuela católica. Los momentos se dividen en tres estándares de preguntas las cuales responderán una finalidad: la primera pregunta es descriptiva, la segunda pregunta de relación y la tercera de interpretación.

Finalmente se desarrollan tres grupos focales, uno en cada colegio con estudiantes de décimo y undécimo grado, en este espacio se recogen las percepciones, voces y posturas de los estudiantes, de cara a las didácticas filosóficas desarrolladas en el aula y la pertinencia de las mismas en la construcción del aprendizaje. En estos grupos focales se presentan tres diferentes tipos de didácticas, que se categorizan como:

- Didáctica del significado: en donde el acento se encuentra en el contenido filosófico de la temática misma. 
- Didáctica del significante: en donde el acento se encuentra en la construcción que realizan los estudiantes alrededor de un problema.

- Didáctica del Significado, en donde el docente propicia una acción que repercute en una reflexión, relacionándola con el contenido teórico.

El desarrollo del grupo focal permite después la socialización de la experiencia a partir de algunas preguntas problematizadoras, recogiendo la percepción de los estudiantes que se consolidará en el dato por analizar, así entonces el grupo focal, "se diferencia de otras formas de entrevista grupales, en cual el GF es focalizado, es decir se centra sobres estímulos o situaciones externas de interés del investigador y es relativamente organizado por un moderador" (Páramo, 2008, p. 149).

En lo que respecta al contexto y los sujetos de la investigación, Bogotá es una ciudad en crecimiento, que asiste a la transformación de una mentalidad local, a una perspectiva cosmopolita en donde se reúne una pluralidad de culturas, costumbres, valores y representaciones provenientes de todos los rincones del país, que llegan a la capital por múltiples causas, formando así un inmenso mosaico urbanístico, que trae consigo la riqueza multicultural, pero también un sin fin de retos y problemáticas que afectan el ser y el actuar de los que habitan la capital colombiana.

En este horizonte la Iglesia de Bogotá se encuentra en un estado de cuestionamiento, por medio del cual se replantea su misión frente a las circunstancias actuales del contexto urbano y social de la ciudad, este rasgo específico determina la búsqueda de la escuela católica en la ciudad de Bogotá cuyo componente específico es "ver la ciudad como obra humana, que responde a la vocación profunda del ser humano de vivir con otros" (Arquidiócesis de Bogotá, 2013, p.14).

Así pues la escuela católica en Bogotá, se encuentra unida al replanteamiento de la misión que ejerce la Iglesia en la ciudad, buscando dentro de su misión específica ser luz del mundo y sal de la tierra. Es así como el campo educativo se convierte en un areópago de evangelización y de diálogo entre la fe, la filosofía y la cultura. Específicamente la Zona pastoral del Espíritu Santo busca responder desde la escuela a las diferentes problemáticas sociales que aquejan a este sector de la ciudad desde el mismo momento de su fundación el 26 de agosto de 1959, 
cuando la unión parroquial del sur, buscó socorrer la marginalidad y la pobreza marcadas por la violencia creciente en el país y las continuas migraciones hacia la ciudad.

Esta zona que comprende el sur de la ciudad desde la Caracas hasta la autopista sur; y desde la av. Primera hasta lo más alto de los cerros de ciudad bolívar, se encuentra fortificada con la riqueza de diferentes instituciones educativas de confesión católica y más específicamente de identidad diocesana, que han encontrado en la educación, el fenómeno crucial para la formación de una nueva persona que genere una nueva sociedad.

Dicha unión escolar conforma lo que se ha denominado como colegios parroquiales y los cuales han tomado una nueva identidad llamada Sistema Educativo Arquidiocesano de Bogotá (SEAB). La búsqueda de un criterio en común en el horizonte evangelizador de la arquidiócesis, ha conducido al Sistema Educativo Arquidiocesano, hacia "aquella unidad que impide la dispersión por las varias ramas del saber y del aprendizaje y que mantiene en el centro a la persona en su compleja identidad, trascendencia e historia" (Congregación para la educación católica, 1997, $\mathrm{N}^{\circ} 10$ ). Esta articulación escolar tiene como punto común la orientación del Proyecto de vida, entendiendo que "la persona de cada uno, en sus necesidades materiales y espirituales, es el centro del magisterio de Jesús: por esto el fin de la escuela católica es la promoción de la persona humana" (Juan Pablo II, 1991, p. 4)

Dentro de estos sujetos institucionales se encuentran las personas que participan en esta investigación, divididas en dos grandes grupos. Por un lado, se encuentran los tres docentes titulares del aprendizaje de filosofía de las instituciones participantes, ellos orientan este aprendizaje desde los pre-saberes y los conocimientos adquiridos en su formación profesional. Su presencia en esta investigación es fundamental ya que aporta la comprensión del ejercicio didáctico y la ejecución de la práctica pedagógica en filosofía, dentro de la Escuela Católica.

Por otro lado, se encuentran los estudiantes quienes ejercen el co-protagonismo del proceso didáctico al proporcionar los datos de comprensión filosófica y descripción de la práctica pedagógica que realizan los docentes. Estos grupos están conformados por hombres y mujeres pertenecientes a grado décimo y grado undécimo de las instituciones participantes, entre los quince y los dieciocho años. El número de integrantes de cada grupo es de cinco, para un total de quince estudiantes. 


\section{Discusión y análisis de los resultados}

En este capítulo se realiza la interpretación de los datos recogidos en el trabajo de campo, los cuales presentan la experiencia de encuentro con los docentes titulares del aprendizaje y los estudiantes participantes del grupo focal. El lector encontrará tres lógicas de lectura, por un lado se encuentra la comparación que arrojan los datos entre las perspectivas docentes institucionales; por otro lado se presentan las posturas de los estudiantes frente a la didáctica de la filosofía Finalmente se realiza una comparación al interior de las instituciones generando líneas de trabajo y temas de investigación.

Se realizó un análisis de comprensión entorno a la problemática didáctica del aprender filosofía o aprender a filosofar y sobre las rutas metodológicas emprendidas por los docentes en la clase de filosofía. Para ello se estudió la posición de los docentes frente a las mismas en cada una de las instituciones, junto a las comprensión que tienen sus estudiantes; los datos recogidos corresponden a una entrevista semiestructurada que presenta las posturas del docente; por otro lado, en el trabajo con los estudiantes al interior del grupo focal, se presentaron tres conclusiones generadas entorno a las didácticas estudiadas y a las sugerencias que ellos realizaron para mejorar la clase de filosofía.

Un primer momento de reflexión se encontró enfocado en la didáctica y la filosofía, las cuales gozan de una autonomía no excluyente, que supone un eje articulador que es la búsqueda de sentido del aprendizaje filosófico en la escuela, este se adquiere gracias a dos componentes: el significado que son los contenidos teóricos y el significante que es el sujeto de aprendizaje. Estos han generado la problemática didáctica sobre el enseñar filosofía o el aprender a filosofar, la cual dio a esta investigación una orientación sobre el desarrollo de esta materia en la escuela. Las posiciones de los docentes entrevistados frente a esta problemática fueron las siguientes: 


\begin{tabular}{|c|c|c|}
\hline $\begin{array}{l}\text { El Docente del Instituto San } \\
\text { Ignacio de Loyola (ISAILO) }\end{array}$ & $\begin{array}{l}\text { El Docente del Instituto } \\
\text { Tecnológico del Sur (FITS) }\end{array}$ & $\begin{array}{l}\text { El Docente del Inmaculado } \\
\text { Corazón de María (Incodemar) }\end{array}$ \\
\hline $\begin{array}{l}\text { "Para mí se aprende a } \\
\text { filosofar, no se trata de dar un } \\
\text { contenido vacío o sin sentido } \\
\text { para el estudiante, sino que } \\
\text { el estudiante sea capaz de } \\
\text { confrontarse frente a lo que } \\
\text { yo le estoy proponiendo, a } \\
\text { fin de generar un cambio de } \\
\text { conducta significativo para su } \\
\text { propia vida". }\end{array}$ & $\begin{array}{l}\text { "Hoy en día y máximo si } \\
\text { es filosofía, no debe ser un } \\
\text { pensamiento extremo, cuando } \\
\text { vamos con los extremos los } \\
\text { resultados no son positivos. } \\
\text { Desde mi experiencia tomo } \\
\text { de las dos, me fundamento } \\
\text { mucho desde el punto de vista } \\
\text { argumentativo y teórico, de } \\
\text { pronto un poquito hegeliano, } \\
\text { pero busco la forma de que } \\
\text { estos contenido no queden ahí, } \\
\text { que se dé un cambio de actitud, } \\
\text { la filosofía no debe quedarse en } \\
\text { lo teórico por lo teórico" }\end{array}$ & $\begin{array}{c}\text { "La filosofía no puede } \\
\text { desconocer lo teórico, pero } \\
\text { antes debe iluminar el contexto } \\
\text { para darse cuenta que la } \\
\text { pregunta complementa la } \\
\text { respuesta. }\end{array}$ \\
\hline
\end{tabular}

Tabla 1. Aparte de la entrevista a docentes.

Los tres docentes presentaron posiciones diferentes con relación a la problemática didáctica de la filosofía, sin embargo, dichas posiciones subyacen a una visión instrumental de la didáctica, la cual polariza la comprensión misma de la filosofía como un aprendizaje escolar. Dentro de los estudios didácticos recientes una visión de diálogo entre ambas posturas abre las puertas al debate, conciliando la problemática en un círculo de comprensión análogo:

Ningún profesor de filosofía puede iniciar a sus estudiantes en la reflexión filosófica sin informarle sobre los problemas, conceptos y términos propios de la filosofía occidental que se han ido constituyendo a lo largo de la historia. El sentido kantiano de aprender a filosofar, se complementa con el sentido hegeliano histórico-crítico de aprender filosofía. (Gómez, 2003, pp. 18-19)

Los docentes han coincidido en una concepción pragmática sobre la didáctica, en donde la ven como un medio o un instrumento para lograr un fin educativo, pero no la han asumido como una disciplina independiente de reflexión sobre el propio aprendizaje, lo cual la reduce a un objetivo instrumental. El punto de divergencia se encuentra en la causa final de la didáctica, la cual no busca facilitar un aprendizaje, sino darle un sentido, a partir de la reflexión que hace sobre el aprendizaje mismo. La búsqueda de un sentido, como fundamento epistemológico propio de la didáctica, supone que ésta no sea equiparada a la filosofía misma, lo cual indica que un campo de trabajo para optimizar el desarrollo de la filosofía como una materia escolar y como una práctica pedagógica, es el desarrollo de una 
diferencia entre la disciplina filosófica como un aprendizaje escolar, y la didáctica como una ciencia autónoma.

La no comprensión del papel de la didáctica en la filosofía, conduce a la problemática de la polarización del sentido, que ha reafirmado el significado, o ha centralizado al significante. Una didáctica analógica de la filosofía, no se centraría en el significado de los contenidos filosóficos, ni en el significante de la producción de los estudiantes; sino en el sentido que se da al aprendizaje filosófico, partiendo de los contenidos e impacto la vida. Esto es lo que constituye una filosofía vitalmente objetiva, que habla a la realidad del estudiante, pero no se queda en el bagaje de la doxa, sino que permite en el lenguaje y la socialización la construcción de una episteme, capaz de exteriorizar la subjetividad de los significantes, sin excluir la objetividad de los significados. En este momento se puede decir que el aprendizaje filosófico ha tenido un sentido didáctico.

La problemática con relación al aprendizaje de la filosofía o del filosofar, atañe no solamente a los docentes, sino también a los estudiantes pues ellos son protagonistas y sujetos significantes en el ejercicio de comprensión y aplicación del pensamiento filosófico.

Laclase defilosofíacomo una materiaescolar, respondea las necesidades vitales propias de los sujetos que participan en ella. Sus expectativas, sus proyecciones y subjetividades adquieren un peso fundamental en la apropiación del sentido del conocimiento. Sin embargo, dicho ejercicio se encuentra condicionado por el paradigma didáctico que el docente ha proyectado en sus estudiantes. En los grupos focales se trabajaron tres tipos diferentes de didácticas, al final los estudiantes manifestaron las mejoras que quisieran darle a su clase sentando las siguientes posiciones:

\begin{tabular}{|c|c|c|}
\hline Grupo Focal Isailo & Grupo Focal FITS & Grupo Focal Incodemar \\
\hline $\begin{array}{l}\text { “Los cinco estudiantes } \\
\text { coinciden en sugerir la } \\
\text { implementación de una } \\
\text { didáctica hermenéutica, que } \\
\text { les permita comprender los } \\
\text { postulados filosóficos a partir } \\
\text { de la experiencia. Sin embargo } \\
\text { hacen énfasis en que es } \\
\text { necesaria la complementación } \\
\text { con las otras didácticas". }\end{array}$ & $\begin{array}{c}\text { "Dentro de las mejoras a la clase } \\
\text { hubo una principal insistencia en } \\
\text { la implementación de la didáctica } \\
\text { hermenéutica en la clase y de } \\
\text { una interacción de las didácticas. } \\
\text { De igual manera los estudiantes } \\
\text { afirman que su clase es teórico } \\
\text { conceptual, pero que su docente } \\
\text { hace de esta didáctica un espacio } \\
\text { ameno y cercano que les permite } \\
\text { aprender". }\end{array}$ & $\begin{array}{l}\text { "Cuatro de los cinco estudiantes } \\
\text { coinciden en llevar a la práctica } \\
\text { los contenidos teóricos por medio } \\
\text { de la didáctica hermenéutica, } \\
\text { a fin de poder comprender la } \\
\text { aplicación de los mismos a la } \\
\text { vida. De igual manera se sugiere } \\
\text { no dejar de lado el componente } \\
\text { teórico, sino que ven necesario } \\
\text { poderlo debatir en la didáctica } \\
\text { activa a fin de que ellos puedan } \\
\text { aprender a expresarse con } \\
\text { argumentos". }\end{array}$ \\
\hline
\end{tabular}

Tabla 2. Aparte de los datos Grupos Focales 
Los aprendizajes escolares, buscan responder a las necesidades propias de los niños, adolescentes y jóvenes que se forman en la escuela, a fin de que estos mismos sean empleados en la consolidación de personas científicamente formadas, capaces de aplicar en su vida, aquello que asumieron como propio en la escuela y que forjaron la propia personalidad. Así entonces el estatuto didáctico propio del aprendizaje filosófico no busca la acumulación de significados o contenidos teóricos; tampoco desea condensar un cumulo de opiniones subjetivas. Su finalidad es suscitar pensamiento que aporte a la formación de una personalidad estable.

Enseñar al alumno a pensar es, fundamentalmente, ayudarle a formar su personalidad, entendiendo por ésta la afirmación de lo que el individuo es según la auténtica libertad y responsabilidad. La filosofía está basada en el diálogo auténtico, en sentido socrático, que no es solo el intercambio de opiniones, sino la respetuosa actitud de búsqueda de una verdad común. Este diálogo, además, debe constituir una apertura hacia la historia del espíritu. (Arpini, 1988, p. 9)

Al aplicar tres estilos didácticos diferentes: el teórico, el activo y el hermenéutico, los estudiantes, han coincidido en afirmar que a sus clases de filosofía les hace falta una didáctica hermenéutica que les enseñe a interpretar los significados teóricos con situaciones concretas, las cuales les permitan adquirir un criterio desde el donde comprender su vida misma, las realidades sociales que les rodean, su relación ecológica con el ambiente y la formación de una personalidad racional, madura y critica.

Los estudiantes no excluyen el fundamento teórico de los conceptos o la producción activa de sus opiniones, por el contrario los consideran necesarios dentro de la clase de filosofía. El vacío se encuentra en que no saben cómo llevar a su cotidianidad lo aprendido en el aula, lo cual les ha creado el paradigma inmediatista en donde se aprende para ser evaluado, y se olvida lo aprendido; o en donde se expone la opinión bajo el estatuto de la libertad de expresión donde todo se considera válido, pero no se vislumbra una verdad consensuada, pensada y discutida.

Una interpretación de conjunto, permitió comprender que la problemática Didáctica de las vertientes hegeliana y kantiana, es asumida por los docentes desde la perspectiva del diálogo, sin embargo los estudiantes al interior de los grupos focales aún evidencian que en la práctica del aula, se da una centralidad a la dimensión teórica y a la 
apropiación de contenidos conceptuales propios de la materia, teniendo como objetivo un estilo de evaluación teórico-conceptual como lo propone la Prueba Saber.

Hay un interés por generar una didáctica en diálogo, pero se evidencia la polaridad entre el contenido teórico conceptual y la propuesta crítica activa, debido al no conocimiento de un puente que les permita generar una interpretación de las mismas. Las tres posturas docentes, encuentran divergencias con las expectativas que tienen sus estudiantes con relación al desarrollo didáctico del aprendizaje. El docente del Isailo, ha realizado una apuesta por la postura del aprender a filosofar, sin embargo sus estudiantes reclaman los contenidos teóricos y su interpretación, por medio de una didáctica hermenéutica que acompañe la didáctica activa de su clase.

Aunque el docente del FITS afirma la necesidad de una lógica del diálogo en el problema didáctico, tanto él como sus estudiantes coinciden en que la clase le da una primacía al componente teórico, el cual no ha sido problema porque los estudiantes lo evidencian como "ameno", sin embargo, reclaman una didáctica que permita llevar ese contenido a la vida diaria. Por otra parte, el docente del Incodemar habla de una enseñanza contextualizada que no olvide los contenidos, por su parte los estudiantes reafirman la necesidad de una didáctica hermenéutica que permita llevar los contenidos a la contextualización, lo cual indica que la clase carece del componente interpretativo.

Teniendo claro que el objetivo de la didáctica es encontrar el sentido del aprendizaje por medio del significado (componente teórico) y el significante (sujeto de aprendizaje), un campo de trabajo es optimizar la relación de interpretación y comprensión que los estudiantes hacen de los contenidos teóricos al aplicarlos a su vida, para lo cual un buen instrumento es la didáctica hermenéutica que pone en diálogo el concepto con la experiencia y su aplicación en la cotidianidad, por medio de un ejercicio comprensivo de interpretación.

Un segundo punto a tratar dentro de la problemática didáctica de la filosofía, es el que gira en torno a las metodologías filosóficas empleadas al interior de las clases, las cuales trazan una ruta de trabajo hacia la apropiación del conocimiento y su aplicación en la cotidianidad de los estudiantes, estos métodos dinamizan el ejercicio académico al interior del aula, los docentes de las instituciones emplean métodos diversos dentro de su práctica pedagógica, así lo expresan ellos: 


\begin{tabular}{|c|c|c|}
\hline $\begin{array}{l}\text { El Docente del Instituto San } \\
\text { Ignacio de Loyola (Isailo) }\end{array}$ & $\begin{array}{l}\text { El Docente del Instituto } \\
\text { Tecnológico del Sur (FITS) }\end{array}$ & $\begin{array}{l}\text { El Docente del Inmaculado } \\
\text { Corazón de María (Incodemar) }\end{array}$ \\
\hline $\begin{array}{l}\text { "Buscamos aprendizaje } \\
\text { colaborativo, aprendizaje } \\
\text { significativo, formas de } \\
\text { trabajo en grupos, debates, } \\
\text { acercamiento a textos } \\
\text { continuos o discontinuos, que } \\
\text { el estudiante se exprese con } \\
\text { argumentos y se atreva a } \\
\text { escribir". }\end{array}$ & $\begin{array}{l}\text { “el método didáctico se centra } \\
\text { en la reflexión del estudiante } \\
\text { desde la participación, } \\
\text { argumentación y explicación } \\
\text { de los contenidos. Que el } \\
\text { estudiante pueda realizar una } \\
\text { reflexión constante y un cambio } \\
\text { de actitudes”. }\end{array}$ & $\begin{array}{l}\text { "El diseño de pensamiento es } \\
\text { una estrategia que se realiza } \\
\text { en marketing y que se aplica en } \\
\text { pedagogía, este diseño ayuda a } \\
\text { formular respuestas prácticas al } \\
\text { entorno". }\end{array}$ \\
\hline
\end{tabular}

Tabla 3. Aparte de entrevista a docentes.

Son múltiples y variados los métodos didácticos que se ofertan en las instituciones educativas, sin embargo, la problemática de los mismos no es de forma sino de fondo, ya que no es la arandela de una estructura la que produce el conocimiento; más bien es la cercanía con la realidad del estudiante la que suscita su aprehensión. Así entonces el método didáctico deja de ser una herramienta, para convertirse en un ejercicio de asimilación con la propia vida: "La elección concreta de la forma de conocimiento debe ser informada por el principio de precaución, la preferencia debe ser dada a la forma de conocimiento que garantice mayor nivel de participación a los grupos sociales involucrados en su diseño" (De Sousa, 2010, p. 56).

Los docentes emplean diversos métodos que se centran en la adquisición de contenido teóricos, su preocupación o sus propósitos de aprendizaje tienden a la teorización y al dominio de un saber; sin embargo, no es suficiente ofertar métodos que llenen las necesidades teórico-conceptuales de un sistema de evaluación, ya que estos métodos tomarán una distancia de la realidad para divagar en el ideal conceptual. Lo contrario a la metodología de contenido, es el método que busca procesos de conocimiento, no basta con un saber, es necesario procurar un saberhacery un saber-ser. Así entonces la metodología no busca la mejor manera de aprender un tratado filosófico, sino la forma de interpretar problemas de vida cotidianos, desde la reflexión filosófica, a fin de comprender, solucionar, actuar de forma divergente.

Frente al método empleado por sus docentes, los estudiantes de los grupos focales compararon tres sesiones didácticas diferentes, entre las cuales se encuentra la tradicional, la activa y la hermenéutica, al realizar un ejercicio de semejanza con su clase de filosofía, los cinco estudiantes tuvieron la oportunidad de experimentar tres metodologías diferentes 
frente a las cuales actuaron de diversas maneras. Se observó su posición frente a cada metodología confrontando con cuál aprendía más y cuál le generaba un aprendizaje para la vida, esta observación suscito los siguientes datos presentes en el diario de campo:

\begin{tabular}{|c|c|c|}
\hline Grupo Focal Isailo & Grupo Focal FITS & Grupo Focal Incodemar \\
\hline $\begin{array}{l}\text { “Tres de los cinco estudiantes } \\
\text { respondieron, que habían } \\
\text { tenido un mayor aprendizaje } \\
\text { con la didáctica Teórico } \\
\text { Conceptual; dos con la } \\
\text { Didáctica Hermenéutica y } \\
\text { ninguno con la didáctica } \\
\text { Activa. Aunque la mayoría } \\
\text { de los estudiantes afirma } \\
\text { aprender más con la } \\
\text { Didáctica Teórico Conceptual, } \\
\text { solamente un estudiante } \\
\text { aprobó en su totalidad la } \\
\text { Prueba Parcial de esta } \\
\text { didáctica. Con relación al } \\
\text { aprendizaje para la vida, tres } \\
\text { estudiantes afirman que la } \\
\text { dinámica Hermenéutica les } \\
\text { deja un mayor aprendizaje } \\
\text { para su vida; un estudiante } \\
\text { afirman que es la Didáctica } \\
\text { Teórico-Conceptual; y uno } \\
\text { afirma que la didáctica Activa } \\
\text { le deja un mayor aprendizaje } \\
\text { para la vida”. }\end{array}$ & $\begin{array}{l}\text { "Tres de los cinco estudiantes } \\
\text { respondieron, que habían } \\
\text { tenido un mayor aprendizaje } \\
\text { con la didáctica Teórico } \\
\text { Conceptual; dos con la Didáctica } \\
\text { Hermenéutica y ninguno con } \\
\text { la didáctica Activa. Aunque la } \\
\text { mayoría de los estudiantes } \\
\text { afirma aprender más con la } \\
\text { Didáctica Teórico Conceptual, } \\
\text { solamente un estudiante aprobó } \\
\text { en su totalidad la Prueba } \\
\text { Parcial de esta didáctica. Con } \\
\text { relación al aprendizaje para la } \\
\text { vida, tres estudiantes afirman } \\
\text { que la dinámica Hermenéutica } \\
\text { les deja un mayor aprendizaje } \\
\text { para su vida; dos estudiantes } \\
\text { afirman que la Didáctica Teórico- } \\
\text { Conceptual les deja un mayor } \\
\text { aprendizaje para su vida; y } \\
\text { ninguno afirma que la didáctica } \\
\text { Activa les deje un mayor } \\
\text { aprendizaje para la vida". }\end{array}$ & $\begin{array}{c}\text { “Uno de los cinco estudiantes } \\
\text { respondió, que había tenido } \\
\text { un mayor aprendizaje con la } \\
\text { didáctica Teórico Conceptual; } \\
\text { dos con la Didáctica } \\
\text { Hermenéutica y dos con la } \\
\text { didáctica Activa. Solamente } \\
\text { un estudiante aprobó } \\
\text { completamente la prueba teórico } \\
\text { conceptual propuesta. Con } \\
\text { relación al aprendizaje para la } \\
\text { vida, dos estudiantes afirman } \\
\text { que la dinámica Hermenéutica } \\
\text { les deja un mayor aprendizaje } \\
\text { para su vida; un estudiante } \\
\text { afirman que la Didáctica Teórico- } \\
\text { Conceptual les deja un mayor } \\
\text { aprendizaje para su vida; y dos } \\
\text { afirman que la didáctica Activa } \\
\text { les deja un mayor aprendizaje } \\
\text { para la vida”. }\end{array}$ \\
\hline
\end{tabular}

Tabla 4. Aparte de los datos Grupos Focales.

Las propuestas metodológicas desarrolladas en la clase de filosofía se pueden dividir en tres grupos, por un lado, los métodos heteroestructurantes que se centran en la conceptualización, como lo es el caso de la cátedra magistral la cual "Es una presentación unidireccional porque el centro del trabajo está en el discurso del maestro, está preparado en cada paso y los alumnos sólo intervienen dependiendo de las ideas expuestas por el docente" (MEN, 2010, p. 114).

Por otro lado se encuentran los métodos auto-estructurantes, en donde se le da prioridad a la posición personal de un estudiante frente a un tema y su argumentación en una comunidad debatiente así "la Filosofía en un trabajo que, aunque puede dar sus frutos finales en el pensamiento de cada uno, es un trabajo de investigación que madura en el diálogo con los demás" (MEN, 2010). Finalmente está el método analógico-hermenéutico: 
Los textos pueden ser escritos, hablados y actuados, como el que se da en el salón de clases. Pero lo común en la hermenéutica es la búsqueda de la comprensión de algo que se toma como un texto, y ese texto es puesto en su contexto, con lo cual se da la interpretación. (Beuchot, 2010, p.18)

Un dato de interés para esta investigación es encontrar que siete de los quince estudiantes entrevistados, respondieron que el método teórico conceptual era con el que más aprendían, frente a seis que se inclinaron por el método hermenéutico y dos por el método activo del debate. Al aplicar una prueba conceptual tipo ICFES, solamente dos estudiantes aprobaron en su totalidad la prueba. Estos resultados varían cuando el aprendizaje se proyecta a la vida, así entonces ocho de los quince estudiantes se inclinaron por el método hermenéutico, mientras cuatro permanecieron con el método teórico, y tres con el debate activo.

Este análisis de los resultados muestra que los estudiantes se han acostumbrado a una metodología de tipo teórica, la cual les ha habituado a un modo de aprender, esto lo ha indicado la primera pregunta que se les realizo con relación al método que les había generado un mayor aprendizaje, por tal motivo presentan una "Zona de Confort" ${ }^{6}$ ante el primer estilo de didáctica teórico-conceptual, sin embargo a la hora de evaluar los conceptos dados según el modelo de esta didáctica, los resultados no son satisfactorios.

Esto ha sido una tendencia en los colegios visitados, el hecho de que los estudiantes se acostumbran a aprender de una sola manera. Al proponer un método como el de la didáctica activa o de debate, los estudiantes no se abrieron al aprendizaje, pues se centraron en la defensa o exposición de una postura personal, pero no dieron el paso de la opinión al conocimiento. Finalmente, en la pregunta por el aprendizaje para la vida, se supondría que deberían coincidir los resultados con relación a la primera pregunta, pero hay un giro en el cual los estudiantes privilegian el método hermenéutico el cual era novedoso para ellos.

Frente a la problemática metodológica en los estudiantes, ha habido un impacto mayor sobre el método hermenéutico, con relación a los métodos implementados por los docentes titulares, esto lo evidencia las sugerencias que realizan para mejorar sus clases, sin embargo para que

6. Cuando se habla de Zona de Confort se hace referencia a un espacio, contenido o modo de aprendizaje en el que el estudiante se siente seguro, pues está acostumbrado a él. 
pueda darse el método hermenéutico es necesario el sustento teórico (significado) y la participación activa de los estudiantes (Significante), a fin de generar un conocimiento aplicado a la vida (Sentido). Por tal motivo, los estudiantes no evidencian un rechazo hacia los otros métodos, sino que recalcan la complementariedad de los mismos y la necesidad de que los tres estilos didácticos puedan desarrollarse en la clase de filosofía. Un campo de trabajo es la búsqueda de ejes articuladores entre los métodos hetero-estructurantes, auto-estructurantes y analógicos.

\section{Una hermenéutica analógica da la flexibilidad para tener no una sola interpretación como válida, según lo hace la hermenéutica univocista, ni tampoco innúmeras interpretaciones, todas válidas y complementarias; sino varias interpretaciones, pero jerarquizadas de modo que unas se acerquen más a la verdad textual y ostras se alejan de ella. (Beuchot, 2009, p. 17)}

Al Interpretar estos datos en conjunto y en referencia institucional se evidencia que los tres docentes tienen diversas comprensiones metodológicas, sin embargo sus estudiantes las asimilan de una manera diferente al ideal docente, y las relacionan con la categoría teóricoconceptual y la perspectiva activa de debate, lo cual indica que los métodos en cuanto a su forma son diferentes, pero en su esencia están buscando responder a un fin teorético, y es de esta manera como el estudiante asume el ejercicio que realiza su profesor.

El docente del ISAILO promueve un método centrado en el desarrollo de diferentes ejercicios académicos que buscan alcanzar la adquisición de una visión filosófica de la vida, la cual se centra en la argumentación activa del filosofar; sin embargo los estudiantes perciben la metodología como un ejercicio teórico, el cual contrario al objetivo del profesor dificulta la disertación activa del debate, así se observó en el grupo focal donde los estudiantes tuvieron mayor resistencia a la didáctica activa, a la cual su docente le da prioridad metódica.

El docente del FITS coincide con sus estudiantes al identificar el método teórico-conceptual como el empleado en las clases de filosofía, dicho método no presenta una posición de apatía por parte de los estudiantes, por el contrario, es evaluado como un ejercicio importante para el desarrollo conceptual, sin embargo la centralidad del método impide la participación total de los estudiantes, la innovación a la hora de experimentar y de llevar a la cotidianidad lo visto en el aula de clase. 
El docente del Incodemar, tiene tras su método de Diseño de pensamiento, un fin pragmático que permita encontrar soluciones útiles a problemas específicos, sin embargo, sus estudiantes lo perciben desde una perspectiva teórica, existe en ellos un presupuesto teórico y una fuerte tendencia a teorizar. ¿Qué tanto este método está procurando una dinámica de participación consiente de los estudiantes? Ciertamente el método es dinámico, sin embargo, el presupuesto de teorización de los estudiantes reduce el método a la adquisición de una teoría no participante.

Hay una diversidad de metodologías en los ejercicios didácticos de la clase de filosofía, lo cual reafirma la inexistencia de un horizonte metodológico en el aprendizaje de filosofía, que lo determina como una materia escolar no unificada, sino asumida desde las diversas ópticas institucionales o docentes. Estas variadas ofertas impiden la articulación de este aprendizaje en la concepción de un Sistema Educativo unificado, y en la interlocución académica de estudiantes procedentes de diversas instituciones. Nuevamente sale a la luz de esta investigación la necesidad de unificar un criterio curricular para la filosofía como una materia escolar, permitiendo con ello que "la escuela se convierte en una instancia de redefinición y reconstrucción social de las culturas locales" (Herrera, 2017, p. 18)

\section{Conclusiones}

La filosofía escolar ha sido reglamentada por la legislación educativa colombiana, sin embargo aún no es claro el lugar que tiene este aprendizaje dentro de la mega estructura de una educación estandarizada que educa para la eficacia y no para la divergencia, motivo por el cual son múltiples y variadas las comprensiones que se realizan al interior de las instituciones educativas, las cuales ejercen una autonomía sobre este aprendizaje llevándolo incluso a diluirse en los currículos o en las filosofía institucionales que se describen en los PEI.

Ciertamente existen unas orientaciones sobre el aprendizaje filosófico, las cuales no son conocidas por la totalidad de los docentes, quienes ejercen las prácticas pedagógicas en filosofía con la comprensión que cada uno de ellos tiene sobre el aprendizaje, pero careciendo de un horizonte común, que permita construir metas y competencias filosóficas de sentido, capaces de tocar transversalmente a todas las instituciones 
educativas que orientan el aprendizaje escolar de filosofía.

Al interrogarse esta investigación sobre la pertinencia de este aprendizaje en la etapa escolar, encontró que la filosofía como un aprendizaje escolar tiene un fin propio que responde a la natural tendencia del hombre por el conocimiento, esta condición de posibilidad inscrita en la mente del ser humano, abre las puertas a la ejercitación filosófica sistemática desde la formación escolar media y vocacional, teniendo claro que la semilla de la inquietud racional nace con la más temprana interrogación del hombre en sus primeros años de vida. La presencia de este aprendizaje dentro de los planeas curriculares de la escuela secundaria se sustenta en el fin mismo de procurar una educación integral que le permita al estudiante construir una identidad, que en el fondo es un conocimiento de sí mismo, de sus semejantes y de su entorno.

La Filosofía entonces tiene un lugar dentro de la formación escolar de las nuevas generaciones, y su causa final es la de orientar al hombre hacia el pensarse a sí mismo por medio de la pregunta, la reflexión, la interpretación y la crítica que nace de su capacidad racional. Justificar este papel ante la legislación colombiana, atañe a los mismos docentes de filosofía que deben hacer un ejercicio de metacognición sobre sus prácticas pedagógicas a fin de observar con mirada crítica el desarrollo mismo de la clase de filosofía en el encuentro con los estudiantes y el impacto vital que puede suscitar en ellos.

La problemática del "¿para qué?" conduce a esta investigación a reflexionar sobre el cómo, el cual responde a las mediaciones didácticas empleadas por el docente posibilitando con ello un aprendizaje significativo. El primer problema encontrado al reflexionar sobre la didáctica, es el de la instrumentalización de esta disciplina, la cual es puesta al servicio pragmático de la filosofía, reduciendo la didáctica al hacer. La didáctica se reconoce como una ciencia cuyo estatuto epistemológico es independiente al de la filosofía, pues busca hacer reflexivo el trabajo que la filosofía hace sobre sí misma, posibilitando su enseñanza en un contexto determinado de aprendizaje. Es allí donde surge la diferencia radical entre la filosofía como una ciencia humana y la filosofía como un aprendizaje escolar.

La didáctica filosófica, entra en un debate de desarrollo al encontrarse con dos posiciones, por un lado, la perspectiva hegeliana que procura 
la enseñanza teórica de la filosofía, comprendida en una línea histórica. Por otro lado, se encuentra la visión kantiana que procura el aprendizaje del filosofar, acentuando el esfuerzo sobre la producción del estudiante. Ambos extremos son nocivos para la formación escolar de una persona, ya que uno procura la teorización de un contenido lejano a la vida misma; y el otro propone la exaltación de la doxa, de la opinión sobre la Episteme. El Ministerio de Educación Nacional en sus orientaciones se ha inclinado más por el proceso kantiano del aprender a filosofar.

De igual manera los docentes reconocen la importancia de no pisar cualquiera de las orillas, sin embargo el dato más recurrente y de acuerdo común de todos los estudiantes participantes, fue el de identificar que sus clases se centraban en la teorización de una didáctica hegeliana. Hay claridad en la necesidad de una didáctica dialógica, sin embargo la misma comprensión conceptual de las figuras evaluadoras externas ha llevado a cambiar la forma de la didáctica con múltiples nombres, aunque perdure el problema de fondo que es la parcialización en una de las orillas del problema didáctico filosófico, aunque sea maquillado con un creativo adjetivo o apelativo conceptual.

Al hacerse una aproximación al fin mismo de la didáctica, se descubre que la didáctica busca dar sentido al aprendizaje, y para que la construcción de sentido pueda suscitarse, son necesarios dos pilares insustituibles. Por un lado, se encuentra el significado, que es el contenido teórico y conceptual de la ciencia a estudiar. Por otro lado, está el significante, que es el sujeto, la persona que aprende, el cual está cargado de su subjetividad y de los interrogantes que le atañen. Tanto significado como significante son necesarios para que se suscite el sentido, de allí que la finalidad de la didáctica filosófica es poner en diálogo de interpretación tanto los contenidos del significado, como la subjetividad del significante. Los estudiantes aprendieron más para la vida con una didáctica hermenéutica dialógica, que con las didácticas que favorecían la parcialización de significados y significantes. 


\section{Referencias bibliográficas}

Acevedo Zapata, S. (2014). Inclusión digital y educación inclusiva. Aportes para el diseño de proyectos pedagógicos con el uso de tecnologías de la comunicación. Revista De Investigaciones UNAD, 13(1), 41-57. doi:http://dx.doi.org/10.22490/25391887.1130

Acevedo Zapata, S. (2015). La formación de profesionales desde la reflexión en la acción con elementos fundamentales de la pedagogía crítica. Revista De Investigaciones UNAD, 14(1), 151 - 169. doi:http://dx.doi.org/10.22490/25391887.1350

Amaya Balaguera, Y. (2015). Guía metodológica ágil, para el desarrollo de aplicaciones móviles "AEGIS-MD". Revista De Investigaciones UNAD, 14(1), 97 - 113.

Ameigeiras, A. (2006). El abordaje etnográfico en la investigación social. En I. Vasiliachis, I. (coord.).(2009). Estrategias de investigación cualitativa. (pp. 107-149). Barcelona: Gedisa.

Arpini, A. (1988). Orientaciones para la enseñanza de la filosofía en el nivel medio. Buenos Aires: El Ateneo.

Arquidiócesis de Bogotá. (2013). Plan de Evangelización 2013 a 2022.

Bogotá: ISPA.

Beuchot, M. (2009). La hermenéutica analógica en Filosofía. En RIIEP. 2(1). pp. 13-22

Beuchot. M. (2010). Hermenéutica analógica, educación y filosofía. Bogotá: Universidad Santo Tomas.

Congregación para la educación católica (1997). La escuela católica en los umbrales del tercer milenio. Roma. Disponible en: http://www.vatican.va/roman_curia/ congregations/ccatheduc/index_sp.htm

De Sousa Santos, B. (2010). Descolonizar el saber, reinventar el poder. Montevideo: Ediciones Trilce.

Gómez Mendoza, M.A. (2003). Introducción a la didáctica de la filosofía. Pereira: Editorial Pampiro.

Herrera, D. (2016). La relación escuela-comunidad: un análisis desde la teoría de sistemas a nueve experiecias en América Latina. En RIIEP. 9(1). pp.11-33.

Jiménez Mendoza, W., Soto Carrión, C., \& Urrutia Huamán, R. (2015). Relación entre modelo PIHEM y gestión educativa en instituciones educativas del nivel inicial en el distrito de Abancay Apurímac Perú, 2014. Revista De Investigaciones UNAD, 14(1), 81 95. doi:http://dx.doi.org/10.22490/25391887.1347 
Juan Pablo II (1991). "Discurso al primer Convenio Nacional de la Escuela Católica en Italia". L'Obsservatore Romano. 24, XI.

Lurán Rivero, A., Buenahora Tobar, M., \& Vargas Granados, C. (2015). Perspectiva del aprendizaje tradicional y del aprendizaje con comunicación bidireccional en actividades educativas. Revista De Investigaciones UNAD, 14(1), 253-262. doi:http://dx.doi.

org/10.22490/25391887.1468

Ministerio de Educación Nacional (2010). “Orientaciones Pedagógicas para la Filosofía en la Educación Media”. Doc. N¹4, Bogotá: Revolución Educativa Colombia Aprende.

Páramo, P. (2008). La investigación en las ciencias sociales. Bogotá: Universidad Piloto de Colombia.

Ricœur, P. (2000). Del texto a la acción. Buenos Aires: Fondo de Cultura Económica.

Rockwell, E. (1999). Etnografía y teoría de la investigación educativa. En M. Balderrama, et al. La investigación etnográfica aplicada a la educación. (pp. 15-27). Bogotá:

Dimensión Educativa.

Rojas Mesa, J., \& Leal Urueña, L. (2014). Entre flujos y fronteras: la educación superior mediada tecnológicamente vista a través de una perspectiva etnográfica. Revista De Investigaciones UNAD, 13(2), 9-27. doi:http://dx.doi.org/10.22490/25391887.1143

Silva Carreño, W., \& Beltrán Martin, J. (2015). El rol de género como fundamento humanista de la formación para la ciudadanía. Revista De Investigaciones UNAD, 14(1), 7 - 17. doi:http://dx.doi.org/10.22490/25391887.1343

Vasilachis, I. (2006). La investigación cualitativa. En I Vasilachis, et al., Estrategias de investigación cualitativa. (pp. 23-60). Barcelona: Gedisa. 